\title{
Putting Differentials Back into Calculus
}

\author{
Tevian Dray \\ Department of Mathematics \\ Oregon State University \\ Corvallis, OR 97331 \\ tevian@math.oregonstate.edu
}

\author{
Corinne A. Manogue \\ Department of Physics \\ Oregon State University \\ Corvallis, OR 97331 \\ corinne@physics.oregonstate.edu
}

June 18, 2009

\begin{abstract}
We argue that the use of differentials in introductory calculus courses provides a unifying theme which leads to a coherent view of calculus. Along the way, we will meet several interpretations of differentials, some better than others.
\end{abstract}

\section{Introduction}

... many mathematicians think in terms of infinitesimal quantities: apparently, however, real mathematicians would never allow themselves to write down such thinking, at least not in front of the children.

Bill McCallum [16]

Calculus is rightly viewed as one of the intellectual triumphs of modern civilization. Mathematicians are also justly proud of the work of Cauchy and his contemporaries in the early 19th century, who provided rigorous justification of the methods introduced by Newton and Leibniz 150 years previously.

Leibniz introduced the language of differentials to describe the calculus of infinitesimals, which were later ridiculed by Berkeley as "ghosts of departed quantities" [4]. Modern calculus texts mention differentials only in passing, if at all. Nonetheless, it is worth remembering that calculus was used successfully during those 150 years. In practice, many scientists and engineers continue to this day to apply calculus by manipulating differentials, and for good reason. It works. The differentials of Leibniz [15] — not the weak imitation found in modern texts - capture the essence of calculus, and should form the core of introductory calculus courses. 


\section{Practice}

The preliminary terror ... can be abolished once for all by simply stating what is the meaning - in common-sense terms — of the two principal symbols that are used in calculating. These dreadful symbols are:

(1) $d$, which merely means "a little bit of". ...

(2) $\int$ which is merely a long $S$, and may be called ... "the sum of".

Silvanus P. Thompson [26]

\subsection{Differentials}

Differentials are commonly used informally when making substitutions in integrals. For example, the power law in the form

$$
d\left(u^{2}\right)=2 u d u
$$

together with the substitution $v=u^{2}$ leads to

$$
2 \int e^{u^{2}} u d u=\int e^{v} d v=e^{v}=e^{u^{2}}
$$

Differentials are equally effective for differentiation.

How many times have you seen a student claim that the derivative of $x^{2}+y^{2}$ is $2 x+2 y$, or even that the derivative of $\pi^{2}$ is $2 \pi$ ? Using differentials naturally eliminates such errors. Equation (1) above is nothing but the power rule in differential language, and leads immediately to

$$
d\left(x^{2}+y^{2}\right)=2 x d x+2 y d y
$$

which could lead to implicit differentiation by "dividing" by $d x$, or to related rates by "dividing" by $d t$. Manipulations such as these are typical of the way in which scientists and engineers use calculus in practice, but are rarely taught in mathematics classes. And even for the student who fails to recognize immediately that $\pi^{2}$ is a constant, the use of differentials leads to

$$
d\left(\pi^{2}\right)=2 \pi d \pi
$$

a completely correct, if perhaps misleading, assertion.

A striking feature of this usage of differentials is that it is not necessary to specify the independent variable. In (2), one does not need to know whether $y$ is a function of $x$ or vice versa, or whether both are functions of some other variable, such as time. Equation (1) is not a statement about derivatives, at least not yet. Rather, it is a statement about the relative rates of change of $u^{2}$ and $u$, regardless of what other quantities $u$ may depend on.

For another example, consider the volume of a cylinder, given by $V=\pi r^{2} h$. The product rule in the form

$$
d(u v)=v d u+u d v
$$

leads immediately to

$$
d V=2 \pi r h d r+\pi r^{2} d h
$$


without first having to decide which of $V, r$, and $h$ are changing, or whether the problem seeks the rate of change of one of these quantities with respect to radius, time, temperature, or possibly something else. Equation (4) is equally valid for related rates problems ("divide" by $d t$ ) or for constrained optimization (set $d V=0$ and solve for $\frac{d h}{d r}$ - or perhaps $\frac{d r}{d h}$ ).

It is important to realize, however, that the differential itself is never the answer to any physical question. Rather, it is the key which unlocks several questions: "Dividing" by $d t$ tells you how quickly the volume is increasing - per second (say), with the correct units readily apparent. Even if one regards (4) as nothing but a heuristic tool, it has clear advantages as a problem-solving strategy. Why clutter this up with assertions such as $r=f(t)$ ? Mathematicians may think that way, but few of our colleagues in other disciplines do so, nor do most of our students.

Differentials were used in (4) to relate the changes in several quantities which were themselves related through an equation. We will refer to this use of differentials as differentials of equations. We encourage our students to find the differential first, without attempting to solve for one variable in terms of the others.

A quite different notion of differential is obtained by starting with a function $f(x)$ and writing

$$
d f=f^{\prime}(x) d x
$$

We will refer to such differentials as differentials of functions. Unlike differentials of equations, differentials of functions require a choice of independent variable(s) for their definition. In this interpretation, equation (4) must be regarded differently in a related rates problem (with independent variable $t$ ) than in an optimization problem (with independent variable $r$ or $h$ ). Equation (5) also makes clear that, when using differentials of functions, the derivative is the fundamental concept, from which the notion of differentials is derived. Contrast the notion of differentials of functions with the above discussion of differentials of equations, in which differentials come first, and derivatives are obtained by "division".

\subsection{Example}

We present here a more complex problem, solved using differentials, which we believe illustrates both the advantages and disadvantages of this approach.

Consider the problem of minimizing the length of a piecewise straight path connecting two fixed points with a given line, as shown in Figure 1. For instance [17], the line could represent a river along which a single pumping station is to be built to serve two towns. The distances $C, D$, and $S=a+b$ are specified; the goal is to determine $a$ and/or $b$ so that $\ell=p+q$ is minimized.

The standard solution to this problem involves expressing $a, b, p, q$, and hence $\ell$, in terms of a single variable, typically $a$, then minimizing $\ell$ by computing $\frac{d \ell}{d a}$ and setting it equal to zero. This computation is straightforward, but involves the derivatives of square roots and some messy algebra. 


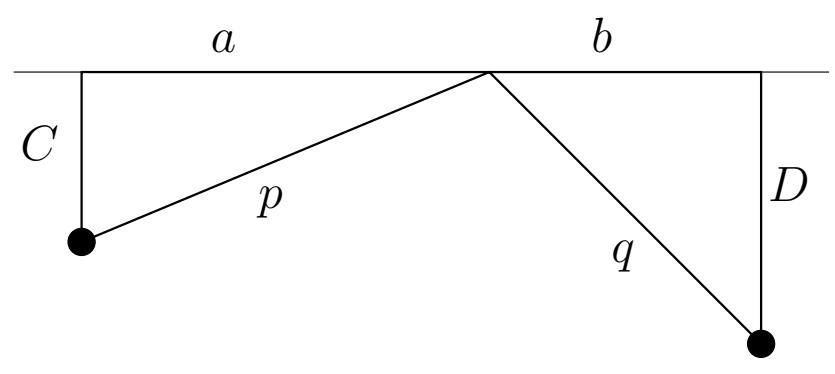

Figure 1: Minimizing the combined distance $p+q$ between two fixed points and a line.

Consider instead the following solution, using differentials. First, write down what you know:

$$
\begin{aligned}
a+b & =S \\
a^{2}+C^{2} & =p^{2} \\
b^{2}+D^{2} & =q^{2} \\
p+q & =\ell
\end{aligned}
$$

where $S, C, D$ are known constants. Next, take the differential of each equation:

$$
\begin{aligned}
d a+d b & =0 \\
2 a d a & =2 p d p \\
2 b d b & =2 q d q \\
d p+d q & =d \ell
\end{aligned}
$$

We are trying to minimize $\ell$, so we set $d \ell=0$ to obtain

$$
0=d \ell=d p+d q=\frac{a}{p} d a+\frac{b}{q} d b=\left(\frac{a}{p}-\frac{b}{q}\right) d a
$$

so that

$$
\frac{b^{2}}{a^{2}}=\frac{q^{2}}{p^{2}}=\frac{b^{2}+D^{2}}{a^{2}+C^{2}}
$$

which (since lengths must be positive) quickly yields

$$
\frac{b}{a}=\frac{D}{C}
$$

so that

$$
a=\frac{C S}{C+D} \quad b=\frac{D S}{C+D}
$$

and it is straightforward to verify that these values do in fact minimize $\ell$.

Is this solution easier than the traditional solution? Maybe. The individual steps, and especially the necessary derivatives, are indeed easier, but there are more of them. Furthermore, there is no single obvious algorithm which tells the student what to do next. The 
differentiation step is straightforward, but solving the resulting system of equations is an art form - and there are several different ways to set up the original equations in the first place.

We regard this "looseness" in the process as a feature, not a bug; students do not learn much from template problem solving. So long as the student writes down correct equations, computes differentials correctly, and keeps substituting one equation into another, the correct solution will eventually emerge.

More importantly, nowhere in the above solution did we specify the independent variable! One could argue that we have implicitly expressed everything in terms of $a$, and in fact a solution involving implicit differentiation would look quite similar. But an equally good case could be made that we instead used $b$, which indicates to us that trying to identify the independent variable is the wrong question to be asking.

We return to this discussion below. 


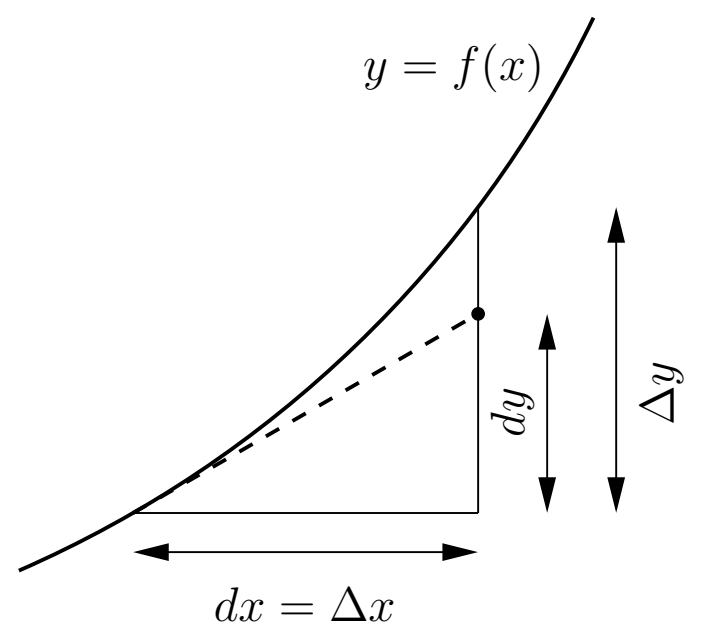

Figure 2: The definition of differential found in many modern textbooks.

\section{Linear Approximation}

Although this may seem a paradox, all exact science is dominated by the idea of approximation.

Bertrand Russell [23]

Modern calculus textbooks introduce differentials - if at all - in the context of linear approximation, as shown in Figure 2. Supposing that $y=f(x)$, the actual change $\Delta y$ in $y$ when changing $x$ by an amount $\Delta x$ can be approximated using the tangent line, so that

$$
\Delta y \approx f^{\prime}(x) \Delta x
$$

The right-hand side is often defined to be $d y$, in which case one would have

$$
d y=f^{\prime}(x) \Delta x
$$

Applying this definition the function $x$, one would also have

$$
d x=\Delta x
$$

and therefore (compare with (5))

$$
d y=f^{\prime}(x) d x
$$

The point of this construction appears to be to distinguish the actual change $\Delta y$ from the approximate change " $d y$ ". In this usage, differentials become just another (finite) variable, which is sometimes emphasized by writing

$$
F(x, d x)=f(x)+f^{\prime}(x) d x
$$

for the linear approximation to $f$ near $x$, although $x$ is usually held fixed so that $F$ can be regarded as a function of the single variable " $d x$ ". 


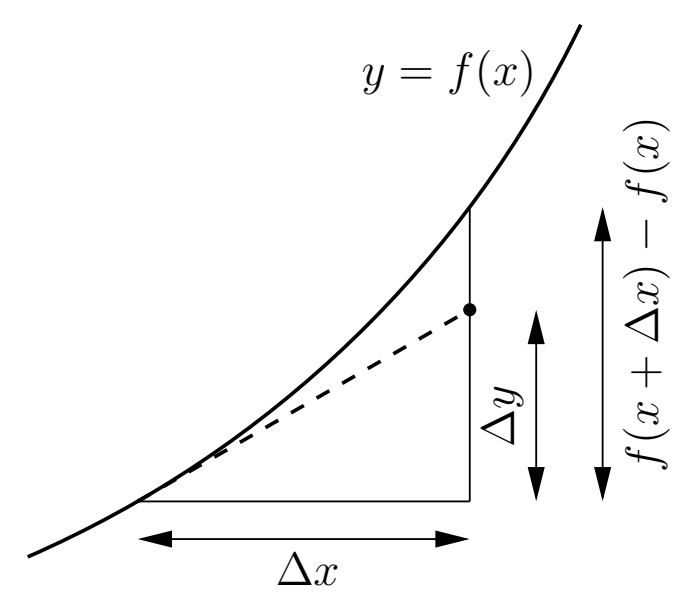

Figure 3: Linear approximation without using differential notation.

One objection to this approach is that it fails to distinguish between the function $f$ and the variable $y$, which only agree on the graph of the function. After all, the goal is to approximate $f$, not $y$. One common variation of the presentation given above avoids this particular difficulty by replacing $d y$ by $d f$ (and $\Delta y$ by $\Delta f$ ). From this point of view, the content of (6) is better expressed as

$$
f(x+\Delta x) \approx f(x)+f^{\prime}(x) \Delta x
$$

which could be written in a form similar to (6) if desired by introducing the notation

$$
\Delta f=f(x+\Delta x)-f(x)
$$

yielding

$$
\Delta f \approx f^{\prime}(x) \Delta x
$$

The linear approximation (9) could then be written quite naturally as

$$
F(x, \Delta x)=f(x)+f^{\prime}(x) \Delta x
$$

with a new variable, such as $h$ or $\varepsilon$, used instead of $\Delta x$ if desired.

A bigger objection to this approach, however, is that we see no good reason to refer to the approximate change as " $d f$ ", let alone " $d y$ ". Nowhere in the argument just given is there a need to use differential notation! Personally, we would use $\Delta y$ to label the approximate change, as shown in Figure 3, since the equation of the tangent line can be naturally expressed as

$$
\Delta y=f^{\prime}(x) \Delta x
$$

However, it's not even obvious that the approximate change needs a label at all. The use of differential notation (" $d y$ ") for this label effectively prevents differentials from being used for any other purpose, and in particular prevents them from being used to describe infinitesimals. (As argued below, that may indeed have been the reason differential notation was adopted for linear approximation.) 
Finally, we point out that the process of linear approximation possesses a fundamental asymmetry between the dependent and independent variables. Nowhere is this more apparent than in Figure 2, in which $d x=\Delta x$, but $d y \neq \Delta y$. It is a useful exercise to draw the analog of Figure 2 for the inverse function $x=f^{-1}(y)$ (assuming it exists), which we encourage the reader to attempt; the resulting notions of $d x$ and $d y$ differ from those in Figure 2. In this sense linear approximation more closely resembles the use of differentials of functions, rather than differentials of equations. 


\section{Theory}

Calculus required continuity, and continuity was supposed to require the infinitely little; but nobody could discover what the infinitely little might be.

Bertrand Russell [24]

Everything is vague to a degree you do not realize till you have tried to make it precise.

Bertrand Russell [22]

\subsection{What are Differentials?}

Our first response to this question is that it doesn't matter. What matters is that ratios of differentials correctly represent derivatives, which they do. We also feel obliged to point out that teachers of introductory students are more likely to care about the answer to this question than their students themselves do.

The usage of differentials survives in most modern calculus courses in the guise of substitutions in integrals, for which computations similar to (1) are commonly used. Why not use them for differentiation as well, as illustrated in Section 2.1?

Mathematicians have been uncomfortable with this response for hundreds of years. While it is not our purpose here to present formal definitions, there are several reasonable definitions of differentials, each of which can be made precise. Among those which we tell our beginning students are that differentials are:

- Arbitrarily small changes in given quantities;

- A shorthand notation for limits;

- Differential forms (see e.g. [10]);

- The infinitesimals of the hyperreal numbers (nonstandard analysis; see e.g. [14]);

- All of the above.

Regardless of which point of view one chooses to adopt, making differentials the fundamental idea on which calculus is based has many advantages. As noted above, they play a key role in substitutions in integral calculus, however much one may feel that this use of differentials is merely heuristic. But the biggest advantages are in differential calculus, where, as outlined in Section 2.1, the use of differentials turns the chain rule, implicit differentiation, and related rates — notoriously difficult concepts for students to master — into something easy rather than hard, organized around a single, coherent idea.

Such advantages are often rediscovered in the literature. For example, Dawson [6] notes that solving related rates problems using implicit differentiation is both easier and less prone to error than substitution, and wonders why constrained optimization problems tend to be solved by substituting the constraint, rather than by what he calls "early differentiation". Using differentials as in the example in Section 2.2, or, as we like to put it, "zapping equations with $d$ ", has all of the benefits of early differentiation, and more - there is no need to specify the independent variable prior to differentiating. Furthermore, the "zapped" equations are 
always linear in the differentials (or, if one prefers, derivatives), and hence can always be solved simultaneously (in well-posed problems).

We now teach every derivative rule in differential form, such as (1) and (3), written in terms of neutral variables such as $u$ and $v$, rather than the overused $x$. Numerous students who first see this approach in our vector calculus classes have commented that they wish they had learned single-variable calculus this way! Students do tend to grumble at first that "it's not in the book", but by the time they master related rates they are usually converted.

\subsection{History}

Arithmetic starts with the integers and proceeds by successively enlarging the number system by rational and negative numbers, irrational numbers, etc... But the next quite logical step after the reals, namely the introduction of infinitesimals, has simply been omitted. I think, in coming centuries it will be considered a great oddity in the history of mathematics that the first exact theory of infinitesimals was developed 300 years after the invention of the differential calculus.

Abraham Robinson [20]

The association of differentials with linear approximation appears to date from the 1940s. (Many of the articles referred to in this section were reprinted in [2].) The earliest discussion of this usage of which we are aware is due to Kac and Randolph [13], who define differentials in terms of derivatives, as in (5), with $d x$ being viewed as a new variable. However, they are careful never to relate $d x$ to $\Delta x$; both are arbitrary. Phipps [18] later attempts to make this usage precise, defining differentials of functions via (7) and (8).

Meanwhile, Church [5] points out the asymmetry between independent and dependent variables, as well as the related crucial role differentials play in integration. Ransom [19] proposes treating equations as fundamental, thus treating all variables equally. An editorial note was inserted at the end of the latter paper by C. B. Allendoerfer, who expresses disagreement with Ransom and solicits further discussion.

Fort [11] emphasizes that the differential is a function of two variables, not only the original independent variable $x$, but also the change $h=\Delta x$ in $x$. This is equivalent to (9) in slightly different notation.

In another editorial comment, Allendoerfer [1] summarizes these and other articles, pointing out that single-variable calculus is fundamentally different from multivariable calculus. He argues in favor of interpreting differentials as a shorthand for a limit argument, claims "no logical connection" between the use of differentials in derivatives and integrals, and assumes that differentials of equations implicitly describe derivatives with respect to a parameter. He concludes,

There is a discredited view that differentials are some sort of "infinitely small quantities." Of course, this is nonsense, but there is a germ of truth in it. For differentials do mark the places in mathematical expressions which are held by small, but finite, quantities while some type of limiting process is carried out in which these small quantities tend to zero. To the writer, they have no other consistent meaning. 
It thus appears that differential notation was appropriated for linear approximation only within the last 60 years, and that one of the motivations for doing so was to "clarify" that infinitesimals are meaningless. This claim was convincingly rebutted by Robinson [20] barely 10 years later, yet little effort has been made to restore the original role of differentials in calculus.

For example, Sylvanus P. Thompson's 100-year-old calculus text [26] — which has never been out of print - uses differentials throughout, treating them as intuitive objects which anyone can master. However, the most recent edition of this text [27] contains a lengthy introduction by Martin Gardner, who effectively undoes this approach by providing modern definitions of functions, limits, and derivatives, alleged prerequisites which the original author clearly chose to do without.

There are, however, some recent attempts to acknowledge that there are viable alternatives to the reinterpretation of differentials in terms of linear approximation. At least one modern calculus text [17] adopts a middle course, being careful never to confuse $d x$ with $\Delta x$, thus at least allowing readers to interpret the former as an infinitesimal if they so choose. Our own interest in differentials is motivated by our successful use of the vector differential $d \overrightarrow{\boldsymbol{r}}$ as the unifying concept in vector calculus [7,9]. We also call the reader's attention to a delightful article [3], which provides an intuitively obvious geometric construction of the hyperreal numbers.

Other disciplines are much more open to the (original) use of differentials. One standard text in electrodynamics [12], a subject which makes heavy use of multivariable calculus, summarizes two years of calculus courses in fewer than 50 pages - using differentials as a matter of course.

\subsection{Differentials in higher dimensions}

Any discussion of the merits of using differentials in calculus should acknowledge that there are some subtleties when using differentials in more than one dimension. For instance, it is natural to write

$$
d A=d x d y=r d r d \phi
$$

for the area element in rectangular and polar coordinates, respectively. (For an explanation of why we use $\phi$ rather than $\theta$, see [8].) However, the second equality requires justification, and only obviously makes sense in the language of differential forms. It is simply not true that $d x d y$ is approximately the same as $r d r d \phi$ for infinitesimal regions, but that is not the point. In order to integrate in higher dimensions, one must first decide how to "chop up" the region. Only then do you know what $d A$ is! At best, the second equality in (10) can be thought of as a convenient shorthand for an equality involving integrals, not just integrands.

We emphasize, however, that there is no problem interpreting differentials as infinitesimals once the decision is made how to integrate; the only problem is in comparing two such choices. And even then, the bottom line is that "equalities" such as (10) always lead to correct answers. 


\section{Conclusion}

But so great is the average person's fear of the infinite that to this day calculus all over the world is being taught as a study of limit processes instead of what it really is: infinitesimal analysis.

Rudy Rucker [21]

We have made two distinct points. First of all, there is a significant difference between differentials of functions and differentials of equations. The student who is able to solve the example in Section 2.2 using differentials has demonstrated a mastery of both calculus and problem-solving skills in a way that a student using traditional methods has not. Furthermore, these same skills can be adapted to virtually any problem in differential calculus, providing a coherence to the subject that may not be obvious to the student in the traditional approach.

Second, there is no need for - and little advantage to - the use of differential notation to describe linear approximation, a usage which is in any case fairly recent. However, their use for this purpose does make it difficult for others to later interpret differentials as infinitesimals - especially for those who favor differentials of equations over differentials of functions. By avoiding the language of differentials when discussing linear approximation, the door would be left open for those who so choose to use differentials to do calculus, with all of the advantages described in Section 2.1.

Science is about physical quantities, not functions. Mathematicians justifiably pride themselves on the development of a rigorous calculus of functions, but it is rather the calculus of physical quantities which is relevant to scientists. Whether one regards differentials as the conceptual core of calculus, as we do, or merely a heuristic tool, they reflect the way many of our professional colleagues think about calculus. This invaluable tool should be emphasized, not relegated to a recently-invented role in linear approximation, for which it is neither suited nor needed.

Surely we should encourage the teaching of a concept which captures the intuitive essence of calculus, which is easy for students to master, and which always produces correct answers.

\section{Acknowledgment}

This work was supported in part by NSF grant DUE-0618877. 


\section{References}

[1] C. B. Allendoerfer, Differentials (editorial), Amer. Math. Monthly 59 (1952) 403-406; reprinted in $[2]$.

[2] T. M. Apostol et al., eds., Selected Papers on Calculus, Mathematical Association of America, Washington DC, 1969.

[3] J. Bair and V. Henry, From mixed angles to infinitesimals, this Journal 39 (2008) $230-233$.

[4] G. Berkeley, The analyst, in From Kant to Hilbert: A Source Book in the Foundations of Mathematics, Vol. 1, William Ewald, ed., Oxford University Press, Oxford, 1996; reprint of pamphlet distributed in 1734; available at http://www.maths.tcd.ie/pub/ HistMath/People/Berkeley/Analyst/Analyst.pdf.

[5] A. Church, Differentials, Amer. Math. Monthly 49 (1942) 389-392; reprinted in [2].

[6] R. Dawson, Differentiate early, differentiate often!, this Journal 36 (2005) 404-407.

[7] T. Dray and C. A. Manogue, The vector calculus gap, PRIMUS 9, (1999) 21-28; available at http://www.math.oregonstate.edu/bridge/papers/calculus.pdf.

[8] - Conventions for spherical coordinates, this JouRnal 34 (2003) 168-169; available at http://www.math.oregonstate.edu/bridge/papers/spherical.pdf.

[9] - Using differentials to bridge the vector calculus gap, this JouRnAL 34 (2003) 283-290; available at http://www . math.oregonstate.edu/bridge/papers/use.pdf.

[10] H. Flanders, Differential Forms with Applications to the Physical Sciences, Academic Press, New York, 1963; reprinted by Dover, Mineola NY, 1989.

[11] M. K. Fort, Jr., Differentials, Amer. Math. Monthly 59 (1952) 392-395; reprinted in [2].

[12] D. J. Griffiths, Introduction to Electrodynamics, 3rd edition, Prentice-Hall, New York, 1999.

[13] M. Kac and J. F. Randolph, Differentials, Amer. Math. Monthly 49, (1942) 110-112; reprinted in $[2]$.

[14] H. J. Keisler, Elementary Calculus: An Infinitesimal Approach, 2nd edition, Prindle, Weber, and Schmidt, Boston, 1982; available at http://www.math.wisc.edu/ $\sim$ keisler/calc.html.

[15] G. Leibniz, New method for maximums and minimums, in A Source Book in Mathematics, 1200-1800, Dirk Jan Struik, ed., Harvard University Press, Cambridge MA, 1969; translation of Nova methodus pro maximis et minimis, pamphlet distributed in 1684. 
[16] W. G. McCallum, review of Calculus Mysteries and Thrillers by R. G. Woods and How To Ace Calculus: The Streetwise Guide by C. Adams, J. Haas, and A. Thompson, Amer. Math. Monthly 108 (2001) 90-93.

[17] W. McCallum, D. Hughes-Hallett, A. Gleason, et al., Calculus, 4th edition Wiley, Hoboken NJ, 2005.

[18] C. G. Phipps, The relation of differential and delta increments, Amer. Math. Monthly 59 (1952) 395-398; reprinted in [2].

[19] W. R. Ransom, Bringing in differentials earlier, Amer. Math. Monthly 58 (1951) 336337 ; reprinted in [2].

[20] A. Robinson, Nonstandard Analysis, Proc. Royal Academy of Amsterdam, A64 (1961) $432-440$.

[21] R. Rucker, Infinity and the Mind: the Science and Philosophy of the Infinite, Princeton University Press, Princeton NJ, 1995.

[22] B. Russell, The Philosophy of Logical Atomism, University of Minnesota, 1949; reprinted by Open Court Publishing, Chicago, 1985.

[23] - quoted in W. H. Auden and L. Kronenberger, eds., The Viking Book of Aphorisms, Viking Press, New York, 1966.

[24] - , quoted in N. Rose, Mathematical Maxims and Minims, Rome Press, Raleigh NC, 1988.

[25] H. M. Schey, Div, Grad, Curl and all that, 4th edition Norton, New York, 2005.

[26] S. P. Thompson, Calculus Made Easy, Macmillan, London, 1910.

[27] S. P. Thompson and M. Gardner, Calculus Made Easy, St. Martin's Press, New York, 1998 